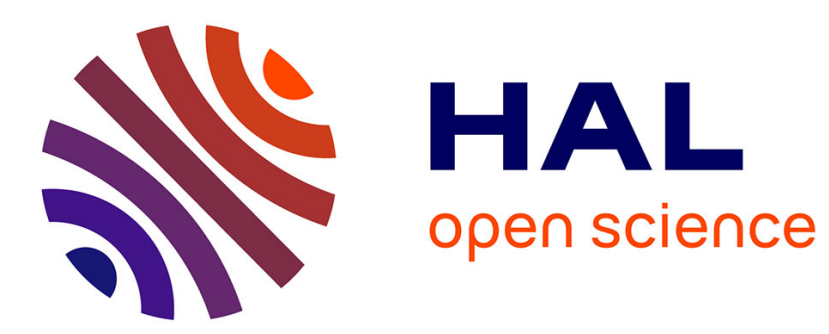

\title{
A quantitative preference-based structured argumentation system for decision support
}

Nouredine Tamani, Madalina Croitoru

\section{To cite this version:}

Nouredine Tamani, Madalina Croitoru. A quantitative preference-based structured argumentation system for decision support. FUZZ-IEEE, Jul 2014, Beijing, China. pp.1408-1415, 10.1109/FUZZIEEE.2014.6891601 . lirmm-01089588

\section{HAL Id: lirmm-01089588 https://hal-lirmm.ccsd.cnrs.fr/lirmm-01089588}

Submitted on 2 Dec 2014

HAL is a multi-disciplinary open access archive for the deposit and dissemination of scientific research documents, whether they are published or not. The documents may come from teaching and research institutions in France or abroad, or from public or private research centers.
L'archive ouverte pluridisciplinaire HAL, est destinée au dépôt et à la diffusion de documents scientifiques de niveau recherche, publiés ou non, émanant des établissements d'enseignement et de recherche français ou étrangers, des laboratoires publics ou privés. 


\title{
A quantitative preference-based structured argumentation system for decision support
}

\author{
Nouredine Tamani, Madalina Croitoru
}

\begin{abstract}
We introduce in this paper a quantitative preference based argumentation system relying on ASPIC argumentation framework [1] and fuzzy set theory. The knowledge base is fuzzified to allow agents expressing their expertise (premises and rules) attached with grades of importance in the unit interval. Arguments are then attached with a strength score aggregating the importance expressed on their premises and rules. Extensions, corresponding to subsets of consistent arguments, are also attached with forces computed based on their strong arguments. The forces are used then to rank extensions from the strongest to the weakest one, upon which decisions can be made. We have also shown that the strength preference relation defined over arguments is reasonable [2] and our fuzzy ASPIC argumentation system can be seen as a computationally efficient instantiation of the generic model of structured argumentation framework introduced in [2].
\end{abstract}

\section{INTRODUCTION}

A RGUMENTATION is a reasoning process which can help making a decision by handling conflicting situations expressed within a discussion among participants (or agents) having different goals and objectives. Dung's abstract framework for argumentation [7] is made of a set of arguments and a binary relation defining attacks between arguments. According to this attack relation, subsets of consistent arguments, called extensions, can be built corresponding to coherent arguments that also defend themselves against attacks. From application standpoint, when arguments reflet agents' knowledge, we need to express argument structure and attack relations to be able to implement the reasoning underlying the argumentation process. In this context, due to its generic nature, the abstract model can be logically instantiated by defining arguments based on a logical language and binary attack relations relying on negation.

In logically structured argumentation systems such as ASPIC/ASPIC + frameworks [1], [2], arguments involve premises (simple facts such as "the weather is rainy") and rules (such as material implications of the form "if it is raining, then I have to take an umbrella"). Premises and rules can be either strict (knowledge which cannot be contradicted or attacked) or defeasible (knowledge that can be attacked or put into question). An argument is defined recursively such that premises are basic arguments, which allow the application of rules having these premises as antecedents. The application of a rule delivers a new conclusion, which in its turn triggers off new rules, and so on. The process goes on through the set of rules to build all arguments

Nouredine Tamani and Madalina Croitoru are with the Graphik team at LIRMM: Laboratoire d'Informatique, de Robotique et de Microlectronique de Montpellier, 161 Rue Ada, 34090 Montpellier, France (email: \{tamani, croitoru\}@lirmm.fr). of the system. This kind of argumentation systems is also equipped with a contrariness function which can be seen as a generalization of the negation, used to define attack relations between arguments. So, logically instantiated argumentation systems, provide means (i) to express argument as a combination of premises and inference rules, (ii) to define contradictions, attacks and defeat between arguments and (iii) to extract the extensions, upon which decision can be made. But in practice, the decision-maker has often to deal with several extensions leading to conflicting decisions. The argumentation process can then help understanding the justification behind a decision but it cannot say what is the best extension to consider to make it.

To address this issue, several approaches for defining preference-based argumentation systems have been proposed during the last years such as [4], [2], [5], [6]. These approaches extend the Dung abstract argumentation model [7] by introducing a preorder or a total order over arguments through a preference relation, which states for each couple of arguments (or even for each couple of subsets of arguments) either they are incomparable or which is the most preferred. In a logically instantiated argumentation framework ([2], [5], [6]), this preference order is used, among other, for a qualitative ranking of extensions. However, in real world application, it can be impossible for the user to specify all the relative importance of arguments, since in the worst case complexity of computation of the ranking is exponential.

Therefore, we propose in this paper a quantitative preference based argumentation system, called F-ASPIC, combining a subset of ASPIC argumentation system [1] and the fuzzy set theory. The crisp notions of strictness and defeasibility are unified under the notion of importance. In this context, the inference rules become more expressive in the sense that the closer to 1 the importance of a rule is, the more significant it is, and conversely the closer to 0 a rule is, the less significant (more defeasible) it is. To ensure the backward compatibility with "crisp" ASPIC argumentation system, we consider any rule attached with degree 1 as strict. Similarly, premises are also attached with a grade of importance defining the fuzzy set of important premises. The closer to 1 the importance of a premise is, the more significant it is, and conversely the closer to 0 a premise is, the less significant it is. Again, to ensure the backward compatibility with "crisp" ASPIC argumentation system, we consider premises attached with degree 1 as axioms.

Arguments have a nested form as in ASPIC argumentation system, but each argument is attached with a quantitative score expressing its strength, computed by using a triangular 
norm (t-norm) aggregating the importance of its involved premises and rules. Based on both negation and argument strength, attacks are also defined so that only arguments having a strength less than 1 can be attacked, and an attack succeed if and only if the attacker is stronger than or is of the same strength as the attacked argument. The extensions delivered from the F-ASPIC argumentation system contain then solid arguments, each of which attached with a grade of strength. The force of each extension is then computed by aggregating the strength of its contained arguments, corresponding to the truth value of the fuzzy quantified proposition "almost_all arguments in the extension are strong", taking into account both the number of arguments and their quality inside an extension. So, we obtain a total order defining an efficient ranking of the extensions in a polynomial time. Finally, we show that (i) the F-ASPIC argumentation system can be seen as a fuzzy extension of ASPIC argumentation system for preference handling, and (ii) F-ASPIC is an efficient instantiation of the preference-based structured argumentation framework (SAF) [2].

The remainder of the paper is organized as follows. In Section II, we recall the principles of argumentation frameworks. In Section III, the rationale behind the fuzzy ASPIC argumentation system is introduced. Fuzzy arguments, their strength, attack and defeat relations, force of extensions and the ranking of extensions are also detailed. In Section IV, we compare our argumentation approach with ASPIC argumentation system and structured argumentation framework. Section V summarizes related work in the field and finally Section VI concludes the paper.

\section{ARguMENTATION FRAMEWORKS}

We recall in this section the abstract argumentation model (Subsection II-A) and the elements of the ASPIC argumentation system (Subsection II-B).

\section{A. Dung argumentation principles}

Dung argumentation framework $(A F)[7]$ is a tuple $(\mathcal{A}, \mathcal{C})$, where $\mathcal{C} \subseteq \mathcal{A} \times \mathcal{A}$ is a binary attack relation on the set of arguments $\mathcal{A}$, having a meaning of defeat.

For each argument $X \in \mathcal{A}, X$ is acceptable w.r.t. a set of arguments $S \subseteq \mathcal{A}$ iff any argument attacking $X$, is attacked by an argument of $S$. A set of arguments $S \subseteq \mathcal{A}$ is conflict free iff $\forall X, Y \in S,(X, Y) \notin \mathcal{C}$. For any conflict free set of arguments $S, S$ is an admissible extension iff $X \in S$ implies $X$ is acceptable w.r.t. $S ; S$ is a complete extension iff $X \in S$ whenever $X$ is acceptable w.r.t. $S ; S$ is a preferred extension iff it is a set inclusion maximal complete extension; $S$ is the grounded extension iff it is the set inclusion minimal complete extension; $S$ is a stable extension iff it is preferred and $\forall Y \notin S, \exists X \in S$ such that $(X, Y) \in \mathcal{C}$.

For $T \in\{$ complete, preferred, grounded, stable $\}, X$ is skeptically (resp. credulously) justified under the $T$ semantics if $X$ belongs to all (resp. at least one) $T$ extension. Output of an extension $E$ is $\operatorname{Concs}(E)=\{\operatorname{Conc}(A), A \in E\}$, where $\operatorname{Conc}(A)$ is the conclusion of argument $A$. The skeptical output of $A F$ is $\operatorname{Output}(A F)=\bigcap_{i=1, \ldots, n} \operatorname{Concs}\left(E_{i}\right)$ such that $E_{i}$ are its $T$ extensions.

\section{B. ASPIC argumentation system}

We consider a subset of ASPIC+ [8], compatible with the one presented in [1]. In the following an ASPIC argumentation system is a tuple $\mathcal{A S}=(\mathcal{L}, c f, \mathcal{R}, \geq)$, where:

- $\mathcal{L}$ is a logical language,

- $c f$ is a contrariness function which associates to each formula of $\mathcal{L}$ a set of its incompatible formulas (in $2^{\mathcal{L}}$ ), it can be considered as a generalization of the negation operator, expressing the contrary and/or the contradiction of formulas,

- $\mathcal{R}=\mathcal{R}_{s} \cup \mathcal{R}_{d}$ is the set of strict $\left(\mathcal{R}_{s}\right)$ and defeasible $\left(\mathcal{R}_{d}\right)$ inference rules of the form $\varphi_{1}, \ldots, \varphi_{m} \rightarrow \varphi$ and $\varphi_{1}, \ldots, \varphi_{m} \Rightarrow \varphi$ respectively, where $\varphi_{i}, \varphi$ are wellformed formulas in $\mathcal{L}$, and $\mathcal{R}_{s} \cap \mathcal{R}_{d}=\emptyset$,

- $\geq$ is a preference ordering over defeasible rules.

A knowledge base in an $A S=(\mathcal{L}, c f, \mathcal{R}, \geq)$ is $\mathcal{K} \subseteq \mathcal{L}$ such that $\mathcal{K}=\mathcal{K}_{a} \cup \mathcal{K}_{p}$ and $\mathcal{K}_{a} \cap \mathcal{K}_{p}=\emptyset, \mathcal{K}_{a}$ contains axioms and $\mathcal{K}_{p}$ contains ordinary premises.

a) ASPIC structured arguments: an ASPIC argument $A$ can have one of the following forms:

1) $\emptyset \rightarrow c$ (resp. $\emptyset \Rightarrow c$ ) with $c \in \mathcal{K}_{a}$ (resp. $c \in \mathcal{K}_{p}$ ) with $\operatorname{Prem}(A)=\emptyset, \operatorname{Conc}(A)=c, \operatorname{Sub}(A)=\{A\}$, $\operatorname{Rules}(A)=\emptyset$, TopRule $(A)=\emptyset$, with Prem returns premises of $A$, Conc returns its conclusion, $S u b$ returns its sub-arguments, Rules returns rules applied on $A$, TopRule returns the last rule applied on $A$,

2) $A_{1}, \ldots, A_{m} \rightarrow c$ (resp. $A_{1}, \ldots, A_{m} \Rightarrow c$ ), such that there exists a strict (resp. defeasible) rule in $\mathcal{R}_{s}$ (resp. in $\mathcal{R}_{d}$ ) of the form $\operatorname{Conc}\left(A_{1}\right), \ldots, \operatorname{Conc}\left(A_{m}\right) \rightarrow c$ (resp. $\left.\operatorname{Conc}\left(A_{1}\right), \ldots, \operatorname{Conc}\left(A_{m}\right) \Rightarrow c\right)$, and $\operatorname{Prem}(A)=$ $\operatorname{Prem}\left(A_{1}\right) \cup \ldots \cup \operatorname{Prem}\left(A_{m}\right), \operatorname{Conc}(A)=c$, $\operatorname{Sub}(A)=\operatorname{Sub}\left(A_{1}\right) \cup \ldots \cup \operatorname{Sub}\left(A_{m}\right) \cup\{A\}$, Rules $(A)=\operatorname{Rules}\left(A_{1}\right) \cup \ldots \cup \operatorname{Rules}\left(A_{m}\right) \cup$ $\left\{\operatorname{Conc}\left(A_{1}\right), \ldots, \operatorname{Conc}\left(A_{m}\right) \rightarrow / \Rightarrow c\right\}$, TopRule $(A)=$ $\operatorname{Conc}\left(A_{1}\right), \ldots, \operatorname{Conc}\left(A_{m}\right) \rightarrow / \Rightarrow c$.

We make the assumption that the set of arguments constructed from $A S$ is finite. An argument is said strict iff it does not involve any defeasible rules or premises. Otherwise, it is called defeasible.

Remark 1. (Notation). An ASPIC argument has a nested form. A sub-argument is also an argument. To improve the readability, by abuse of notation, we associate to each argument a label made of a capital letter followed by a subscript number. The labels are then used in an argument to refer to its sub-arguments. In this notation, a label followed by colon is not a part of the argument.

Example 1. Let $A S$ be an ASPIC argumentation system defining the rules $\mathcal{R}_{s}=\{a, b \rightarrow c\}$ and the ordinary premises $\mathcal{K}_{p}=\{a, b\}$. The following are arguments in $A S$ :

- $A_{1}: \emptyset \Rightarrow a$

- $A_{2}: \emptyset \Rightarrow b$

- $A_{3}: A_{1}, A_{2} \rightarrow c$ 
b) ASPIC attacks and defeat: ASPIC defines rebutting and undercutting attacks.

Rebutting attack. Argument $A$ rebuts argument $B$ iff $\exists A^{\prime} \in \operatorname{Sub}(A): \operatorname{Conc}\left(A^{\prime}\right)=\varphi$ and $B^{\prime} \in \operatorname{Sub}(B)$ such that $B^{\prime}$ is of the form $B_{1}^{\prime}, \ldots, B_{m}^{\prime} \Rightarrow \neg \varphi$. It is defined as a restricted rebut attack [9] to satisfy rationality postulates as shown in [9].

Undercutting attack. Argument $A$ undercuts argument $B$ (or make $B$ inapplicable) iff $\exists B^{\prime} \in \operatorname{Sub}(B)$ of the form $B_{1}^{\prime}, \ldots, B_{m}^{\prime} \Rightarrow \varphi$ and $\exists A^{\prime} \in \operatorname{Sub}(A): \operatorname{Conc}\left(A^{\prime}\right)=$ $\neg\left\lceil\operatorname{Conc}\left(B_{1}^{\prime \prime}\right), \ldots, \operatorname{Conc}\left(B_{n}^{\prime \prime}\right) \Rightarrow \varphi\right\rceil$, with operator $\lceil$.$\rceil con-$ verts a defeasible rule into a literal [10].

Defeat relation. $A$ defeats $B$ if $A$ rebuts or undercuts $B$.

\section{FUZZY ASPIC ARGUMENTATION SYSTEM (F-ASPIC)}

We recall in Subsection III-A elements of the fuzzy set theory. In Subsection III-B, we introduce our fuzzy argumentation system, denoted F-ASPIC, combining the fuzzy set theory and subset of ASPIC structured argumentation framework. F-ASPIC arguments are detailed in Subsection III-C. Fuzzy attack and defeat relations between arguments are defined in Subsection III-D. The rationality postulates are also studied in our fuzzy case in Subsection III-E. The total order between extensions based on the strength of their arguments are finally introduced in Subsection III-F.

\section{A. Fuzzy Set Theory}

Fuzzy set theory is introduced by Zadeh [11] to express the gradual membership of an element to a set. Formally, a fuzzy set $F$ is defined on a referential $U$ by a membership function $\mu_{F}: U \mapsto[0,1]$ such that $\mu_{F}(x)$ denotes the membership grade of $x$ in $F$. In particular, $\mu_{F}(x)=1$ denotes the full membership of $x$ in $F, \mu_{F}(x)=0$ expresses the absolute non-membership and when $0<\mu_{F}(x)<1$, it reflects a partial membership (the closer to $1 \mu_{F}(x)$, the more $x$ belongs to $F$ ). The core of a fuzzy set $F$ is $\operatorname{Core}(F)=\left\{x \in F: \mu_{F}(x)=1\right\}$ and the support of a fuzzy set $F$ is $\operatorname{Support}(F)=\left\{x \in F: \mu_{F}(x)>0\right\}$. A fuzzy set generalizes an ordinary (crisp) set in which membership grades are in $\{0,1\}$. If a fuzzy set is a discrete set then it is denoted $F=\left\{\left(x_{1}, \mu_{F}\left(x_{1}\right)\right), \ldots,\left(x_{n}, \mu_{F}\left(x_{n}\right)\right)\right\}$, otherwise, it is characterized by its membership function, generally a trapezoidal function.

The union $\cup$ and the intersection $\cap$ operators are defined with a couple of a t-norm and a t-conorm, such as (min, max). Let $F, G$ be two fuzzy sets, $\mu_{F \cup G}(x)=$ $\max \left(\mu_{F}(x), \mu_{G}(x)\right), \mu_{F \cap G}(x)=\min \left(\mu_{F}(x), \mu_{G}(x)\right)$, and the complement of $F$, noted $F^{c}$, is defined by $\mu_{F^{c}}(x)=$ $1-\mu_{F}(x)$.

The logical counterparts of $\cap, \cup$ and the complement are resp. $\wedge, \vee$ and $\neg$. Other operators have also been defined such as fuzzy implications [12].

\section{B. Fuzzy argumentation system: F-ASPIC $A S$}

A fuzzy argumentation theory $F A T=(F A S, \mathcal{K})$ is made of a fuzzy argumentation system $F A S$ and a fuzzy knowledge base $\mathcal{K}$. It is equipped with a fuzzy membership function imp expressing for each premise and rule its importance. It is worth noticing that the rules do not model fuzzy implications, but regular implications attached with an importance score.

A fuzzy ASPIC (F-ASPIC) argumentation system is a $F A S=(\mathcal{L}, c f, \mathcal{R}$, imp $)$ such that:

- $\mathcal{L}$ is a logical language,

- $c f$ is a contrariness function (we consider the negation $\neg$ as its basic form),

- $\mathcal{R}$ is the fuzzy set of important rules of the form $\left(\phi_{1}, \ldots, \phi_{m} \rightarrow \phi, s\right)$ with $\phi_{i, i=1, \ldots, m} \in \mathcal{L}$ are the premises of the rule and $\phi \in \mathcal{L}$ is its conclusion, $s \in[0,1]$ is its importance, provided by the experts of the domain. For a given rule $r$, if $\mu_{i m p}(r)=1$ then $r$ is a strict rule, if $\mu_{i m p}(r)=0$ then $r$ is an insignificant rule, discarded by the system. If $\left.\mu_{i m p}(r) \in\right] 0,1[$ then the closer to $1 \mu_{i m p}(r)$ is, the more important $r$ is, and conversely the closer to $0 \mu_{i m p}(r)$ is, the more defeasible $r$ is.

The knowledge base $\mathcal{K}$ defines the fuzzy set of important premises of the form $\left(p, \mu_{i m p}(p)\right)$ :

- if $\mu_{i m p}(p)=1$ then $p$ is an axiom or strict premise,

- if $\mu_{i m p}(p)=0$ then $p$ is an ordinary premise, it can be considered as an insignificant premise, discarded by the system,

- if $\left.\mu_{i m p}(p) \in\right] 0,1\left[\right.$ then the closer to $1 \mu_{i m p}(p)$ is, the more strict (less ordinary) $p$ is, and conversely the closer to $0 \mu_{i m p}(p)$ is, the more ordinary (less strict) $p$ is.

\section{F-ASPIC argumentation system (AS) argument}

A fuzzy ASPIC argument $A$ can have one of the following forms:

1) $\emptyset \rightsquigarrow^{s} c$, with $c \in \mathcal{K}, \operatorname{Prem}(A)=\{c\}, \operatorname{Conc}(A)=c$, $\operatorname{Sub}(A)=\{c\}, \operatorname{Rules}(A)=\emptyset$, TopRule $(A)=\emptyset$, where Prem returns premises of $A$, Conc returns its conclusion, $S u b$ returns its the sub-arguments, Rules returns the rules involved in $A$, TopRule returns the last rule applied to deliver the conclusion $c$, and $s \in$ ]0,1] expresses the strength of $A$ (defined below),

2) $A_{1}, \ldots, A_{m} \rightsquigarrow^{s} c$, such that there exists a rule $r \in$ $\mathcal{R}$ of the form $\left(\operatorname{Conc}\left(A_{1}\right), \ldots, \operatorname{Conc}\left(A_{m}\right) \rightarrow c, s_{r}\right)$, and $\operatorname{Prem}(A)=\operatorname{Prem}\left(A_{1}\right) \cup \ldots \cup \operatorname{Prem}\left(A_{m}\right)$, $\operatorname{Conc}(A)=c, \operatorname{Sub}(A)=\operatorname{Sub}\left(A_{1}\right) \cup \ldots \cup \operatorname{Sub}\left(A_{m}\right) \cup$ $\{A\}, \operatorname{Rules}(A)=\operatorname{Rules}\left(A_{1}\right) \cup \ldots \cup \operatorname{Rules}\left(A_{m}\right) \cup$ $\left\{r^{\prime}\right\}$ with $\left.r^{\prime}=\left(\operatorname{Conc}\left(A_{1}\right), \ldots, \operatorname{Conc}\left(A_{m}\right) \rightarrow c, s_{r}^{\prime}\right)\right\}$, TopRule $=\left\{r^{\prime}\right\}$, and $\left.\left.s \in\right] 0,1\right]$ expresses the strength of $A$ (defined below).

Definition 1. (Strength of an argument). The strength of argument $A$, denoted $\operatorname{str}(A)$, is the strength of its conclusion $(\operatorname{Conc}(A))$ computed as follows:

$\operatorname{str}(A)=\left\{\begin{array}{r}\mu_{i m p}(c), c \in \mathcal{K} \quad \text { if } A \text { is of the form } \emptyset \rightsquigarrow c, \\ T_{e \in R u l e s(A) \cup \operatorname{Prem}(A)}\left(\mu_{i m p}(e)\right) \\ \text { if } A \text { is of the form } A_{1}, \ldots, A_{m} \rightsquigarrow c,\end{array}\right.$

where $T$ is a triangular norm. 
It is worth noticing that the interpretation of this strength differs depending on the used t-norm. The Zadeh t-norm min, for instance, carries a pessimistic vision of the evaluation of an argument strength, because the strength of an argument is the importance of its less important premise or rule. It is also possible to use the probabilistic t-norm $*$, since it can model the principle of parsimony (or economy or Ockham's razor principle) applied on non-strict arguments. So, the more an argument involves more weak rules and premises, the less its strength is.

Definition 2. (Strict/defeasible argument). An argument $A$ is said strict iff $\operatorname{str}(A)=1$. Otherwise, it is called defeasible.

We can define the notion of important argument based on the strength. So, the closer to $1 \operatorname{str}(A)$ the more important argument $A$ is.

Definition 3. (Consistency of $\mathcal{R}$ ). Let $\mathcal{A}$ be a set of arguments, the set of rule $\mathcal{R}$ is said consistent iff $\nexists A, B \in \mathcal{A}$, such that $\operatorname{str}(A)=\operatorname{str}(B)=1$ and $\operatorname{Conc}(A)=\neg \operatorname{Conc}(B)$.

D. Attack and defeat relations between arguments

We define the fuzzy rebut and fuzzy undercut attacks, denoted F-rebut and F-undercut respectively.

Definition 4. ( $F$-rebutting attack). An argument $A F$-rebuts argument $B$ if $\exists A_{i} \in S u b(A)$ and $\exists B_{j} \in S u b(B)$ such that (i) Conc $\left(A_{i}\right)=\neg \operatorname{Conc}\left(B_{j}\right)$, (ii) $\operatorname{str}\left(B_{j}\right)<1$ and (iii) $\operatorname{str}\left(A_{i}\right) \geq \operatorname{str}\left(B_{j}\right)$.

Definition 5. (F-undercuting attack). Argument $A F$ undercuts argument $B$ iff $\exists B^{\prime} \in S u b(B)$ of the form $B_{1}^{\prime}, \ldots, B_{m}^{\prime} \rightsquigarrow^{s} \varphi$, with $s<1$ and $\exists A^{\prime} \in \operatorname{Sub}(A)$ : $\operatorname{Conc}\left(A^{\prime}\right)=\neg\left\lceil\operatorname{Conc}\left(B_{1}^{\prime}\right), \ldots, \operatorname{Conc}\left(B_{m}^{\prime}\right) \rightsquigarrow^{s} \varphi\right\rceil$, with $\operatorname{str}\left(A^{\prime}\right) \geq s$.

The above definitions extend the one introduced in the crisp case [1], in the sense that only defeasible (or less important) argument can be attacked by either a defeasible or a strict argument (or more important).

Definition 6. $(F$-defeat). Argument A F-defeats arguments $B$ iff $A$ F-rebuts or F-undercuts $B$.

\section{E. F-ASPIC AS extensions and rationality postulates}

Rationality postulates, defined in [9], ensure the completeness and the consistency of the output of a logicalbased argumentation system. We consider here the following rationality postulates:

1) Closure under sub-arguments: for every argument in an extension, also all its sub-arguments are in the extension,

2) Closure under strict rules $\mathcal{R}_{s}=\left\{r \in \mathcal{R} \mid \mu_{\text {imp }}(r)=1\right\}$ of the output of an extension: all possible conclusions from applicable strict rules are derived in each extension,

3) Direct consistency: the output of each extension is consistent, so it is not allowed to derive a conclusion and its contradiction in an extension,
4) Indirect consistency: the closure under strict rules of the output of each extension is consistent.

Proposition 1. F-ASPIC AS is closed under subarguments and under strict rules, direct and indirect consistent, iff the set of strict rules $\mathcal{R}_{s}$ is closed under transposition [9] and $\mathcal{R}_{s}$ is consistent.

The proof of Proposition 1 is detailed in the Appendix I. It shows that, based on our definition of attack and defeat relations, extensions can be built upon defeat relation and not on attack relations without falling in the inconsistency described in [5], [6], [2]. So, our logical-based argumentation system is compatible with Dung abstract model for argumentation.

\section{F. Ordering extensions}

In order to rank extensions, we need to define an operator to compute the force of each extension by aggregating the strength of its arguments. Several aggregating operators can be used such as min, $\max$, means operators, etc. Although they are efficient and simple to implement, they can lead to non-intuitive ranking for extensions as shown in the following example.

Example 2. Let $\mathcal{E}_{1}$ and $\mathcal{E}_{2}$ be two extensions, under one Dung semantics, delivered from a F-ASPIC argumentation system, such that:

- $\mathcal{E}_{1}=\left\{\left(A_{1}, 1\right),\left(A_{2}, 0.9\right),\left(A_{3}, 0.3\right),\left(A_{4}, 0.3\right),\left(A_{5}, 0.1\right)\right\}$

- $\mathcal{E}_{2}=\left\{\left(A_{1}^{\prime}, 1\right),\left(A_{2}^{\prime}, 0.9\right),\left(A_{3}^{\prime}, 0.5\right),\left(A_{4}^{\prime}, 0.1\right),\left(A_{5}^{\prime}, 0.1\right)\right\}$ where $\left(A_{i}, s\right)$ (resp. $\left.\left(A_{i}^{\prime}, s^{\prime}\right)\right)$ refers to argument $A_{i}$ (resp. $A_{i}^{\prime}$ ) and its strength $s$ (resp. $s^{\prime}$ ).

The min operator returns force $\left(\mathcal{E}_{1}\right)=\operatorname{force}\left(\mathcal{E}_{2}\right)=0.1$, the $\max$ operator returns $\operatorname{force}\left(\mathcal{E}_{1}\right)=\operatorname{force}\left(\mathcal{E}_{2}\right)=1$, the arithmetic mean returns $\operatorname{force}\left(\mathcal{E}_{1}\right)=\operatorname{force}\left(\mathcal{E}_{2}\right)=0.52$, which state that $\mathcal{E}_{1}$ and $\mathcal{E}_{2}$ are of the same force and interest for decision making. But, we notice that $\mathcal{E}_{2}$ is better than $\mathcal{E}_{1}$, since $\mathcal{E}_{2}$ contains more arguments having a strength of at least 0.5 than $\mathcal{E}_{1}$.

So, we need a hybrid operator able to deliver a force taking into account the strength of arguments and their number. Therefore, we define a force of an extension based on fuzzy quantified proposition as follows.

Definition 7. (Force of an extension). We define the force of an extension E, under one Dung's semantics, as the truth value $\delta_{P}$ of the fuzzy quantified proposition " $P$ : almost_all arguments in E are strong".

$\delta_{P}$ can be efficiently computed in a polynomial time by the decomposition based approach [13]. So arguments in $E$ are ranked from the most to the least strong:

$$
\begin{gathered}
\operatorname{str}\left(A_{1}\right) \geq \operatorname{str}\left(A_{2}\right) \geq \ldots \geq \operatorname{str}\left(A_{n}\right) \text { with } n=|E|, \text { and } \\
\delta_{P}=\max _{i=1, \ldots, n}\left(\min \left(\operatorname{str}\left(A_{i}\right), \mu_{\text {almost_all }}\left(\frac{i}{n}\right)\right)\right)
\end{gathered}
$$

It is worth noticing that the evaluation of $\delta_{P}$ depends heavily on the definition of the linguistic quantifier almost_all. 


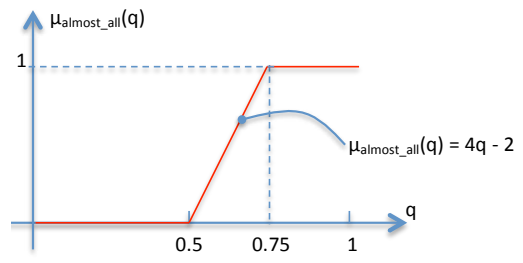

Fig. 1: Example of definition of the quantifier almost_all.

Definition 8. (Ordering relation between extensions). Let FAS be a fuzzy $A S$ and $E_{1}, \ldots, E_{2}$ its extensions under one Dung semantics. The extension $E_{i}$ is preferred than the extension $E_{j}$, denoted $E_{i} \succeq_{e} E_{j}$ iff force $\left(E_{i}\right) \geq \operatorname{force}\left(E_{j}\right)$. Its strict counterpart $\succ_{e}$ is: $E_{i} \succ_{e} E_{j}$ if and only if $\operatorname{force}\left(E_{i}\right)>\operatorname{force}\left(E_{j}\right)$.

Example 3. In the previous example, if the linguistic quantifier is defined as shown in Figure 1, then we get $\operatorname{force}\left(\mathcal{E}_{1}\right)=0.3$ and $\operatorname{force}\left(\mathcal{E}_{2}\right)=0.4$ and $\mathcal{E}_{2} \succeq_{e} \mathcal{E}_{1}$.

\section{COMPARING F-ASPIC WITH ASPIC AND FAS SYSTEMS}

In this section, we show that F-ASPIC can be seen as a fuzzy extension of ASPIC argumentation system (in Subsection IV-A), and F-ASPIC can also considered as a fuzzy instantiation of generic preference-based SAF [2] (in Subsection IV-B).

\section{A. ASPIC AS is a particular case of F-ASPIC AS}

Let $A S$ be a regular ASPIC argumentation system. It is defined on a knowledge base $\mathcal{K}=\mathcal{K}_{p} \cup \mathcal{K}_{a}$ where $\mathcal{K}_{p}$ contains the ordinary premises of the system and $\mathcal{K}_{a}$ contains its axioms. It is easy to obtain from a regular ASPIC $A S$ its corresponding F-ASPIC $A S$ by:

- associating to elements from $\mathcal{K}_{a}$ the degree 1 , and to elements from $\mathcal{K}_{p}$ any score in ]0, 1 [, for instance 0.5 ,

- associating to strict rule the degree 1 , and to defeasible rules any score in $] 0,1[$, for instance 0.5 .

In this case, arguments can either get the strength 1 (strict arguments) or 0.5 (defeasible arguments), and since Fattacks are defined from strict or defeasible arguments to only defeasible arguments then we obtain the same symmetric attacks between defeasible arguments leading to the same extensions.

Conversely, in a F-ASPIC $A S$, when the function $\mu_{i m p}$ associates a same degree (in $] 0,1]$ ) to the premises and a same degree to the rules (in ]0,1]), then the F-ASPIC $A S$ behaves exactly as a regular ASPIC $A S$.

B. Compatibility with the Structured argumentation framework $(S A F)$

1) Structured argumentation framework (SAF) [2]: defined within an argumentation theory $A T=(A S, \mathcal{K})$, is a triple $\langle\mathcal{A}, \mathcal{C}, \preceq\rangle$ where $\mathcal{A}$ is the set of all finite arguments constructed from $\mathcal{K}$ in $A S, \preceq$ is an ordering on $\mathcal{A}$, and $(X, Y) \in \mathcal{C}$ if and only if $X$ attacks $Y$. Moreover:
- A c-SAF is a SAF in which all arguments are required to have a c-consistent set of premises.

- A set $S \subseteq \mathcal{L}$ is c-consistent if for no $\phi$ it holds that $S \vdash \phi, \neg \phi$. Otherwise $S$ is c-inconsistent.

- A SAF is said well-defined if it is (i) axiom consistent, (ii) well-formed [2], and (iii) closed under contraposition or closed under transposition.

Strict continuation of an argument. For any set of arguments $\left\{A_{1}, \ldots, A_{n}\right\}$, the argument $A$ is a strict continuation of $\left\{A_{1}, \ldots, A_{n}\right\}$ if and only if:

- $\operatorname{Prem}_{p}(A)=\bigcup_{i=1}^{n} \operatorname{Prem}_{p}\left(A_{i}\right)$. The ordinary premises in $A$ are exactly those in $\left\{A_{1}, \ldots, A_{n}\right\}$,

- $\operatorname{DefRules}(A)=\bigcup_{i=1}^{n} \operatorname{DefRules}\left(A_{i}\right)$, with $\operatorname{DefRules}(A)$ is a function returning the defeasible rules in $A$. This means that defeasible rules in $A$ are exactly those in $\left\{A_{1}, \ldots, A_{n}\right\}$,

- StRules $(A) \supseteq \bigcup_{i=1}^{n} \operatorname{StRules}\left(A_{i}\right)$ and $\operatorname{Prem}_{n}(A) \supseteq$ $\bigcup_{i=1}^{n} \operatorname{Prem}_{n}\left(A_{i}\right)$, with $\operatorname{StRules}(A)$ and $\operatorname{Prem}_{n}(A)$ are functions returning respectively the strict rules and the axioms of $A$. This means that strict rules and axioms of $A$ are supersets of the strict rules and axioms in $\left\{A_{1}, \ldots, A_{n}\right\}$.

Reasonable argument ordering $\preceq$. An argument ordering is reasonable [2] if and only if:

1) $\forall A, B$, if $A$ is strict and firm and $B$ is defeasible, then $B \prec A$, with $\prec$ is a strict counter-part of $\preceq$,

2) $\forall A, B$ if $B$ strict and firm then $B \nprec A$,

3) $\forall A, A^{\prime}, B$ such that $A^{\prime}$ is a strict continuation of $\{A\}$, if $A \nprec B$ then $A^{\prime} \nprec B$, and if $B \nprec A$ then $B \nprec A^{\prime}$,

4) Let $\left\{C_{1}, \ldots, C_{n}\right\}$ be a finite subset of $\mathcal{A}$, and for $i=1, \ldots, n$, let $C^{+\backslash i}$ be some strict continuation of $\left\{C_{1}, \ldots, C_{i-1}, C_{i+1}, \ldots, C_{n}\right\}$. Then it is not the case that $\forall i, C^{+\backslash i} \prec C_{i}$.

2) Compatibility of FAS and SAF : we can show that the proposed fuzzy argumentation system, the argument ordering $\preceq$ is reasonable.

Definition 9. (argument ordering). Let $A, B$ be two arguments. We define the argument ordering $\preceq$ as $A \preceq B \Leftrightarrow$ $\operatorname{str}(A) \leq \operatorname{str}(B)$. Its strict counter-part is $A \prec B \Leftrightarrow$ $\operatorname{str}(A)<\operatorname{str}(B)$.

Proposition 2. The ordering based on the strength function str is reasonable [2], if the t-norm used is $\mathrm{min}$.

Proof: An argument ordering is reasonable [2] iff

1) Condition 1 holds since a strict argument has a strength of 1 and any defeasible argument has a strength $<1$.

2) Condition 2 also holds, since $\operatorname{str}(B)=1$, then whatever $\operatorname{str}(A)$ we get $\operatorname{str}(B) \nless \operatorname{str}(A)$ and then $B \nprec A$.

3) Condition 3 holds. Indeed, let $x, y, z$ be $\operatorname{str}(A), \operatorname{str}\left(A^{\prime}\right), \operatorname{str}(B)$ respectively. Since $A^{\prime}$ is a strict continuation of $\{A\}$ then $y=T(x, 1)=x$, with $T$ is a t-norm, because a strict continuation involves only strict rules and axioms. 
If $A \nprec B$ then $x \nless z$, but as $y=x$ then $y \nless z$, which means that $A^{\prime} \nprec B$.

If $B \nprec A$ then $z \nprec x$, so $z \nprec y$ and $B \nprec A^{\prime}$.

4) Condition 4 is proved as follows:

Let $C^{+\backslash i}$ be a strict continuation of $\left\{C_{1}, \ldots, C_{i-1}, C_{i+1}, \ldots, C_{n}\right\}$, then $\operatorname{str}\left(C^{+\backslash i}\right)=$ $T_{j=1, \ldots, n \wedge j \neq i} \operatorname{str}\left(C_{j}\right)$, where $T$ is a t-norm.

Let suppose that $\forall i, C^{+\backslash i} \prec C_{i}$, then $\forall i, \operatorname{str}\left(C^{+\backslash i}\right)<$ $\operatorname{str}\left(C_{i}\right) \Leftrightarrow \forall i, T_{j=1, \ldots, n \wedge j \neq i} \operatorname{str}\left(C_{j}\right)<\operatorname{str}\left(C_{i}\right)$, which is not verified for the t-norm $T=\min$, since in this case we should have $\forall C_{i}, \exists C_{j}: \operatorname{str}\left(C_{j}\right)<\operatorname{str}\left(C_{i}\right)$, which is not possible for the weakest argument in any finite subset $\left\{C_{1}, \ldots, C_{n}\right\}$ of $\mathcal{A}$.

Indeed, let $C_{j}$ be the argument such that $\operatorname{str}\left(C_{j}\right)=$ $\min _{\left\{C_{1}, \ldots, C_{n}\right\}} \operatorname{str}\left(C_{j}\right)$. It strict continuation $C^{+\backslash j}$ is based on all other stronger arguments or having the same strength as $C_{j}$ in $\left\{C_{1}, \ldots, C_{j-1}, C_{j+1}, \ldots, C_{n}\right\}$, then $\operatorname{str}\left(C^{+\backslash j}\right) \geq \operatorname{str}\left(C_{j}\right)$ if $T=\min$.

To conclude, the proposed argument ordering based on the t-norm $\min$ is reasonable.

Based on Proposition 2, we can say that our F-ASPIC $A S$ is an efficient instantiation of a SAF model [2], which is easier to implement within a real world application to manage conflicts and to take decision.

\section{RELATED WORK}

Several work have been carried out during the last years to define fuzzy-based argumentation approaches. In [14], [15], [16] a possibilistic approach based on the defeasible logic programming DeLP [17] has been developed. These approaches suffer from the difficulties related to the definition of the necessity measure, since each argument is attached with a necessity degree expressing its certainty. A possibility distribution has also to be considered for the different interpretations or models over the logical language of the system. In addition, theses approaches inherits from the DeLP its possible inconsistencies and incompleteness of the output regarding the rationality postulates, studied in [9]. In the same context, a possibilistic approach has also been proposed in [18]. The considered argumentation structure is based on a fuzzy labeling, as in [19], and a possibility distribution has to be defined over the different interpretations of the logical language. The proposed approach is different from ours in the sense that the used fuzzy information express a reliability of the source of arguments. So, arguments of the same source have a same reliability degree. In our case, a single source can express knowledge of different degrees of importance. In addition, the arguments are not structured, instead of ours in which structured arguments are made of fuzzy premises and rules.

In [20], a fuzzy argumentation system for trust has been proposed. The approach is similar to ours in the spirit but the context is quite different since in this approach arguments are not structured.

Another line has also been investigated in [21], [22] in which the attack relation is fuzzified to express the relative strength of the attack relation between arguments. This approach is rather different than the one proposed in this paper since in our case the attacks still defined as crisp attack. In [21] in addition to the fuzzy representation of attacks, the strength of an attack depends on both the fuzzy set of arguments supporting the attacker and the strength of the attack. In [22], the definition of the defeat relation involves a binary preference relation over arguments, and according to this preference relation attacks can fail. Besides, both approaches consider non-structured arguments. In the same spirit of fuzzifying the attack relation, [23] introduces an argumentation approach based on fuzzy description logic (fuzzy $\mathcal{S H \mathcal { I } F}$ DL). Arguments in this context are a mixture of fuzzy linguistic variables and ontological knowledge. They are related by fuzzy attack and fuzzy support relations. A preference relation is also defined over arguments. This approach does not rely on a structured argumentation framework, which forces the user to manually specify the attack and support relations between concepts.

\section{CONCLUSION}

We have introduced in this paper a quantitative preferencebased argumentation system relying on the fuzzy set theory and ASPIC structured argumentation framework. Arguments are built upon the fuzzy set of important rules and premises, allowing the computation of their strength. Extensions are attached with a score in $] 0,1]$ aggregating the strength of its arguments, based on fuzzy quantified propositions. Finally, we have shown that F-ASPIC $A S$ can be seen as a fuzzy extension of ASPIC $A S$ for preference handling, and the proposed ordering operator between arguments is reasonable and the proposed fuzzy argumentation system is an efficient instantiation of the SAF model [2].

As future work, we plan to implement the approach in a real world application and to perform more tests to study the behavior of the system according to the definition of the linguistic quantifier, and the t-norm used for computing the strength of arguments.

\section{REFERENCES}

[1] L. Amgoud, L. Bodenstaff, M. Caminada, P. McBurney, S. Parsons, H. Prakken, J. Veenen, and G. Vreeswijk, "Final review and report on formal argumentation system.deliverable d2.6 aspic.” Tech. Rep., 2006.

[2] S. Modgil and H. Prakken, "A general account of argumentation with preferences," Artificial Intelligence, vol. 195, pp. 361-397, 2013.

[3] L. Amgoud, J.-F. Bonnefon, and H. Prade, "An argumentation-based approach to multiple criteria decision," in European Conference on Symbolic and Quantitative Approaches to Reasoning with Uncertainty (ECSQARU), 2005, pp. 269-280.

[4] H. Prakken, "An abstract framework for argumentation with structured arguments," Department of Information and Computing Sciences. Utrecht University., Tech. Rep., 2009.

[5] L. Amgoud and S. Vesic, "Repairing preference-based argumentation frameworks," in 21st International Joint Conference on Artificial Intelligence, 2009, pp. 665-670.

[6] _ - "Handling inconsistency with preference-based argumentation," in Proceedings of the 4th international conference on Scalable uncertainty management, ser. SUM'10. Berlin, Heidelberg: Springer-Verlag, 2010, pp. 56-69. [Online]. Available: http://dl.acm.org/citation.cfm?id=1926791.1926805 
[7] P. M. Dung, "On the acceptability of arguments and its fundamental role in nonmonotonic reasoning, logic programming and n-persons games," Artificial Intelligence, vol. 77, no. 2, pp. 321-357, 1995.

[8] H. Prakken, "An abstract framework for argumentation with structured arguments," Argument and Computation, vol. 1, no. 2, pp. 93-124, 2011.

[9] M. Caminada and L. Amgoud, "On the evaluation of argumentation formalisms," Artificial Intelligence, vol. 171, pp. 286-310, 2007.

[10] J. L. Pollock, Cognitive carpentry. A blueprint for how to build a person, M. Cambridge, Ed. MIT Press, 1995.

[11] L. Zadeh, "Fuzzy sets," Information and control, vol. 8, no. 3, pp. 338-353, 1965.

[12] B. Bouchon-Meunier, D. Dubois, L. Godo, and H. Prade, Fuzzy sets in approximate reasoning and information systems, ser. The Handbook of fuzzy sets. Kluwer Academic Publishers, 1999, ch. 1 : Fuzzy set and possibility theory in approximate and plausible reasoning, pp. 27-31.

[13] R. R. Yager, "General multiple-objective decision functions and linguistically quantified statements," International Journal of ManMachine Studies, vol. 21, no. 5, pp. 389-400, 1984.

[14] C. I. Chesñevar, G. R. Simari, T. Alsinet, and L. Godo, "A logic programming framework for possibilistic argumentation with vague knowledge," in Proceedings of the 20th conference on Uncertainty in artificial intelligence, ser. UAI '04. Arlington, Virginia, United States: AUAI Press, 2004, pp. 76-84. [Online]. Available: http://dl.acm.org/citation.cfm?id=1036843.1036853

[15] T. Alsinet, C. I. Chesñevar, L. Godo, and G. R. Simari, "A logic programming framework for possibilistic argumentation: Formalization and logical properties," Fuzzy Sets and Systems, vol. 159, no. 10, pp. 1208-1228, 2008.

[16] T. Alsinet, C. I. Chesñevar, L. Godo, S. Sandri, and G. R. Simari, "Formalizing argumentative reasoning in a possibilistic logic programming setting with fuzzy unification," International Journal of Approximate Reasoning, vol. 48, no. 3, pp. 711-729, 2008. [Online]. Available: http://www.sciencedirect.com/science/article/pii/S0888613X07000977

[17] A. J. García and G. R. Simari, "Defeasible logic programming: an argumentative approach," Theory Pract. Log. Program., vol. 4, no. 2, pp. 95-138, Jan. 2004. [Online]. Available: http://dx.doi.org/10.1017/S1471068403001674

[18] C. da Costa Pereira, A. G. B. Tettamanzi, and S. Villata, "Changing one's mind: Erase or rewind? possibilistic belief revision with fuzzy argumentation based on trust," in Proceedings of the Twenty-Second International Joint Conference on Artificial Intelligence, 2011, pp. 164-171.

[19] C. Gratie and A. Florea, "Fuzzy labeling for argumentation frameworks," in Argumentation in Multi-Agent Systems, ser. Lecture Notes in Computer Science, P. McBurney, S. Parsons, and I. Rahwan, Eds. Springer Berlin Heidelberg, 2012, vol. 7543, pp. 1-8. [Online]. Available: http://dx.doi.org/10.1007/978-3-642-33152-7_1

[20] R. Stranders, M. Weerdt, and C. Witteveen, "Fuzzy argumentation for trust," in Computational Logic in Multi-Agent Systems, ser. Lecture Notes in Computer Science, F. Sadri and K. Satoh, Eds. Springer Berlin Heidelberg, 2008, vol. 5056, pp. 214-230. [Online]. Available: http://dx.doi.org/10.1007/978-3-540-88833-8_12

[21] J. Janssen, D. Vermeir, and M. De Cock, "Fuzzy argumentation frameworks," in International Conference on Information Processing and Management of Uncertainty in Knowledge-Based Systems, 12th, Proceedings, 2008, pp. 513-520.

[22] S. Kaci and C. Labreuche, "Argumentation framework with fuzzy preference relations," in Proceedings of the Computational intelligence for knowledge-based systems design, and 13th international conference on Information processing and management of uncertainty, ser. IPMU'10. Berlin, Heidelberg: Springer-Verlag, 2010, pp. 554-563. [Online]. Available: http://dl.acm.org/citation.cfm?id=1876326.1876395

[23] I. Letia and A. Groza, "Towards pragmatic argumentative agents within a fuzzy description logic framework," in Argumentation in Multi-Agent Systems, ser. Lecture Notes in Computer Science, P. McBurney, I. Rahwan, and S. Parsons, Eds. Springer Berlin Heidelberg, 2011, vol. 6614, pp. 209-227. [Online]. Available: http://dx.doi.org/10.1007/978-3-642-21940-5_13

\section{APPENDIX I}

PROOF OF THE RATIONALITY POSTULATES

Proposition 1. F-ASPIC $A S$ is closed under subarguments and under strict rules, direct and indirect consistent, iff the set of strict rules $\mathcal{R}_{s}$ is closed under transposition [9] and $\mathcal{R}_{s}$ is consistent.

Definition I.1. (closure under sub-arguments). [4] define the closure under sub-arguments as the fact that for every argument in an extension, also all its sub-arguments are in the extension.

Proof: The proof is similar to the one of ASPIC+ [4]. Let $E$ be an extension under one Dung semantics, and suppose $A \in E$ is an argument such that $\exists A_{i}, A_{i} \in$ $\operatorname{Sub}(A) \wedge A_{i} \notin E$.

$A_{i} \notin E \Rightarrow \exists B \in E:$ B attacks $A_{i}$ and $\nexists C \in E$ : $C$ attacks $B$, So $B$ attacks $A$ on $A_{i}$ without being defended by $E$, then $A \notin E$, which is in contradiction with our supposition. Finally, $A_{i} \in E$.

Definition I.2. (closure of a set of formulas under strict rules) Let $\mathcal{P} \subseteq \mathcal{L}$. The closure of $\mathcal{P}$ under strict rules $\mathcal{R}_{s}$, denoted by $C l_{\mathcal{R}_{s}}$, is the smallest set such that:

- $\mathcal{P} \subseteq C l_{\mathcal{R}_{s}}(\mathcal{P})$

- if $\varphi_{1}, \ldots, \varphi_{n} \rightsquigarrow \psi \in \mathcal{R}_{s}$ and $\varphi_{1}, \ldots, \varphi_{n} \in C l_{\mathcal{R}_{s}}(\mathcal{P})$ then $\psi \in C l_{\mathcal{R}_{s}}(\mathcal{P})$.

If $\mathcal{P}=C l_{\mathcal{R}_{s}}(\mathcal{P})$ then $\mathcal{P}$ is closed under the set $\mathcal{R}_{s}$.

Definition I.3. (closure of a $F A S$ under strict rules) Let $F A S=(\mathcal{L}, c f, \mathcal{R}, i m p)$ be a fuzzy argumentation system and $E_{1}, \ldots, E_{n}$ is its extensions under one Dung semantics. The closure under strict rules is defined as follows:

1) $\forall E_{i, i=1, \ldots, n}, \operatorname{Concs}\left(E_{i}\right)=\operatorname{Cl}_{\mathcal{R}_{s}}\left(\operatorname{Concs}\left(E_{i}\right)\right)$,

2) let Output $=\bigcap_{i=1, \ldots, n} \operatorname{Concs}\left(E_{i}\right)$, Output $=$ $\mathrm{Cl}_{\mathcal{R}_{s}}$ (Output).

Proof: The proof is similar to the one introduced in [9]. It is adapted to our context in which rules (strict and defeasible) are fuzzified.

1) Let $E_{i}$ be an extension. We suppose that $\operatorname{Concs}\left(E_{i}\right) \neq$ $C l_{\mathcal{R}_{s}}\left(\operatorname{Concs}\left(E_{i}\right)\right)$.

As $C l_{\mathcal{R}_{s}}\left(\operatorname{Concs}\left(E_{i}\right)\right)$ contains all possible conclusions derived from premises of $E_{i}$ applied on rules of $\mathcal{R}_{s}$, then $\exists r \in \mathcal{R}_{s}$ of the form $\left(\varphi_{1}, \ldots, \varphi_{m} \rightarrow \psi, 1\right)$ such that premises of $r$ are deductible from $E_{i}$ and its conclusion is not in $E_{i}$ :

$\exists A_{1}, \ldots, A_{m} \in E_{i}: \operatorname{Conc}\left(A_{i}\right)=\varphi_{i}$ and $\exists r=$ $\left(\varphi_{1}, \ldots, \varphi_{m} \rightarrow \psi, 1\right) \in \mathcal{R}_{s}$ and $A=A_{1}, \ldots, A_{m} \rightsquigarrow^{s}$ $\psi \notin E_{i}$. We have 2 possible cases:

a) $\exists B \in E_{i},(B$ attacks $A) \vee($ Aattacks $B)$

b) $\exists B \in \mathcal{A}:(B$ attacks $A) \wedge\left(\nexists C \in E_{i}\right.$ : $C$ attacks $B$ ), such that $\mathcal{A}$ is the set of all arguments of the system, which means that $E_{i}$ does not defend $A$.

- Case 1: $\exists B \in E_{i},(B$ attacks $A) \vee($ Aattacks $B)$. - Case 1.1: $\exists B \in E_{i},(B$ attacks $A)$ 
$B$ rebuts $A$ on $A^{\prime} \in S u b(A)$ of the form $\left(A^{\prime}=A_{1}^{\prime \prime}, \ldots, A_{2}^{\prime \prime} \rightsquigarrow^{s^{\prime}} \alpha\right)$ and $\operatorname{str}\left(A^{\prime}\right)<1$, then $\exists B^{\prime} \in S u b(B)$ which contradicts $A^{\prime}$ and $\operatorname{str}(B) \geq \operatorname{str}(A)$. As $B \in E_{i}$ then $\operatorname{Sub}(B) \in$ $E_{i}$ (cf. first rationality postulate), which means that $B$ attacks $A^{\prime}$ and $E$ is then conflictual and cannot be an extension: contradiction.

- Case 1.2: $\exists B \in E_{i},($ A attacks $B)$

In this case, $E_{i}$ must defend $B$ and $\exists C \in E_{i}$ : $C$ attacks $A$ on one of its sub-arguments $A^{\prime} \in E_{i}$ which leads to a conflictual $E_{i}$ and to a contradiction.

- Case 2: $\exists B \in \mathcal{A}:(B$ attacks $A) \wedge\left(\nexists C \in E_{i}\right.$ : Cattacks $B$ ).

$B$ rebuts $A$ on $A^{\prime} \in S u b(A)$ and $A^{\prime} \neq A$ (since $A^{\prime} \in \operatorname{Sub}(A)$ and TopRule $(A)=r$ is a strict rule so $A$ can never attacked by any other argument), then $\exists B^{\prime} \in S u b(B)$ which contradicts $A^{\prime}$. But $A^{\prime} \in E$ then $S u b\left(A^{\prime}\right) \in$ $E_{i}$, which means that $E_{i}$ defends $A^{\prime}$ and so $A$, since $\operatorname{str}\left(B^{\prime}\right) \geq \operatorname{str}\left(A^{\prime}\right)$ and $\operatorname{str}\left(A^{\prime}\right) \leq 1$ then $\operatorname{str}(A)=\min _{A_{i} \in S u b(A)}\left(\operatorname{str}\left(A_{i}\right)\right)$ and $\operatorname{str}(A) \leq$ $\operatorname{str}\left(A^{\prime}\right)$ : contradiction with the supposition.

2) In [9], the authors showed that if $\forall E_{i}, \operatorname{Concs}\left(E_{i}\right)=$ $C l_{\mathcal{R}_{s}}\left(\operatorname{Concs}\left(E_{i}\right)\right)$ then Output $=C l_{\mathcal{R}_{s}}($ Output $)$.

Definition I.4. (consistency of a set of formulas)

Let $\mathcal{P} \subseteq \mathcal{L}$. $\mathcal{P}$ is consistent iff $\nexists \psi, \varphi \in \mathcal{P}$ such that $\psi=$ $\neg \varphi$. Otherwise it is said to be inconsistent.

Definition I.5. (consistency)

Let $F A S=(\mathcal{L}, c f, \mathcal{R}, i m p)$ a fuzzy argumentation system and $E_{1}, \ldots, E_{n}$ is its extensions under one Dung semantics. The direct consistency is defined as follows ([9]):

1) $\forall E_{i, i=1, \ldots, n}, \operatorname{Concs}\left(E_{i}\right)$ is consistent,

2) let Output $=\bigcap_{i=1, \ldots, n} \operatorname{Concs}\left(E_{i}\right)$, Output is consistent.

We show in what follows that if $\mathcal{R}_{s}$ is consistent then $F A S$ satisfies direct consistency.

Proof: The proof is similar to the one detailed in [9].

1) Let $F A S$ be an argumentation system with a consistent $\mathcal{R}_{s}$. Let also $E_{i}$ be an extension and we suppose that $\operatorname{Concs}\left(E_{i}\right)$ is inconsistent. So, $\exists \varphi, \psi \in \operatorname{Concs}\left(E_{i}\right)$ : $\psi=\neg \varphi$, then there exists two arguments $A, B \in E_{i}$ such that:

$\exists A \in E_{i}, A: \varphi_{1}, \ldots, \varphi_{m} \rightsquigarrow^{1} \varphi$ and $\exists B \in E_{i}: B=$ $\psi_{1}, \ldots, \psi_{k} \rightsquigarrow 1 \neg \varphi$.

As $A, B \in E_{i}$ then $A, B$ are not in conflict; so ( $A$ does not attack $B)$ and $(B$ does not attack $A$ ), which means:

$\nexists A^{\prime} \in \operatorname{Sub}(A) \wedge \nexists B^{\prime} \in \operatorname{Sub}(B): \operatorname{conc}\left(A^{\prime}\right)=$ $\neg \operatorname{conc}\left(B^{\prime}\right)$ and $B^{\prime}$ (or $A^{\prime}$ ) is a non-strict argument $\left(\operatorname{str}\left(B^{\prime}\right)<1\right.$ or $\left.\operatorname{str}\left(A^{\prime}\right)<1\right)$, and $\operatorname{str}\left(A^{\prime}\right) \leq \operatorname{str}\left(B^{\prime}\right)$ (or $\operatorname{str}\left(B^{\prime}\right) \leq \operatorname{str}\left(A^{\prime}\right)$ ).

But $A, B$ have contradictory conclusions, then the only case in which that is possible is where $A, B$ are strict arguments, which leads to an inconsistent $\mathcal{R}_{s}$ : contradiction.

2) In [9], the authors showed that if $\forall E_{i}, \operatorname{Concs}\left(E_{i}\right)$ is consistent then Output is also consistent.

Definition I.6. (indirect consistency)

Let $F A S=(\mathcal{L}, c f, \mathcal{R}, i m p)$ an argumentation system and $E_{1}, \ldots, E_{n}$ is its extensions under one Dung semantics. The indirect consistency is defined as follows ([9]):

1) $\forall E_{i, i=1, \ldots, n}, C_{\mathcal{R}_{s}}\left(\operatorname{Concs}\left(E_{i}\right)\right)$ is consistent,

2) let Output $=\bigcap_{i=1, \ldots, n} \operatorname{Concs}\left(E_{i}\right), C_{\mathcal{R}_{s}}($ Output) is consistent.

Proof: In [9], it has been shown that if an argumentation system satisfies closure and direct consistency then it also satisfies indirect consistency. The system has to be able to derive all possible conclusions in each extension (closure of the strict rules) and the set of strict rules must be consistent. Indeed, let $F A S$ be an argumentation system which satisfies closure and direct consistency. As $A S$ satisfies direct consistency then $\forall E_{i, i=1, \ldots, n}, \operatorname{Concs}\left(E_{i}\right)$ and Output are consistent, and as FAS satisfies closure then $\forall E_{i, i=1, \ldots, m}, \operatorname{Concs}\left(E_{i}\right)=$ $C l_{\mathcal{R}_{s}}\left(\operatorname{Concs}\left(E_{i}\right)\right)$ and Output $=C l_{\mathcal{R}_{s}}($ Output $)$, then $\forall E_{i, i=1, \ldots, n}, C l_{\mathcal{R}_{s}}\left(\operatorname{Concs}\left(E_{i}\right)\right)$ and $C l_{\mathcal{R}_{s}}($ Output $)$ are consistent.

It has been shown in [9] that if an $A S$ satisfies closure and direct consistency, it also satisfies indirect consistency. 